\title{
Impact of cosmic inhomogeneities on SNe observations ${ }^{1}$
}

\author{
Kimmo Kainulainen and Valerio Marra \\ Department of Physics, University of Jyväskylä, PL 35 (YFL), FIN-40014 Jyväskylä, Finland and Helsinki \\ Institute of Physics, University of Helsinki, PL 64, FIN-00014 Helsinki, Finland
}

\begin{abstract}
We study the impact of cosmic inhomogeneities on the interpretation of SNe observations. We build an inhomogeneous universe model that can confront supernova data and yet is reasonably well compatible with the Copernican Principle. Our model combines a relatively small local void, that gives apparent acceleration at low redshifts, with a meatball model that gives sizeable lensing (dimming) at high redshifts. Together these two elements, which focus on different effects of voids on the data, allow the model to mimic the concordance model.
\end{abstract}

Keywords: Inhomogeneous Universe Models, Dark Energy, Backreaction, Observational Cosmology PACS: $95.36 .+\mathrm{x}$, 98.62.Sb, 98.65.Dx, 98.80.Es

\section{INTRODUCTION}

Until the nature of the dark energy is completely understood, the "safe" consequence of the success of the concordance model is that the flat isotropic and homogeneous $\Lambda \mathrm{CDM}$ model is a good phenomenological fit to the real inhomogeneous universe and not that the concordance model is the actual model of the universe. It is therefore useful to look for alternative models that fit the data. Here we will discuss the possibility that the late time evidence for dark energy can be explained by the late time formation of nonlinear large-scale inhomogeneities, so that the so-called coincidence problem becomes a hint of new physics.

In the following, we will assume that the spacetime of the inhomogeneous universe is accurately described by small perturbations around the Friedmann-Lemaitre-Robertson-Walker (FLRW) solution whose energy content and spatial curvature are defined as Hubble-volume spatial averages over the inhomogeneous universe. Following Ref. [3] we will call this Hubble-volume average the Global Background Solution (GBS). The cosmological model obtained through observations, on the other hand, will be called the Phenomenological Background Solution (PBS). The idea is to associate the concordance model with the PBS, while the actual GBS is the Einstein-de Sitter (EdS) model.

\section{SETUP}

For consistency with the $\mathrm{CMB}$ spectrum and the age of the universe, the current background expansion rate of the EdS model is taken to be $H_{\infty}=100 h_{\infty} \mathrm{km} \mathrm{s}^{-1} \mathrm{Mpc}^{-1}$ with $h_{\infty}=0.5$. The local expansion rate $H_{0}$ will be higher, about $h \simeq 0.58$ in the current model, which is within $2-\sigma$ of the HST key project [4] value of $0.72 \pm 0.08$ [5].

Our setup is made of two elements. First, we will model the overall universe by a meatball model consisting of two families of randomly placed halos. The family $A$ describes very large, low density contrast structures and the family $B$ models large clusters of galaxies. The parameters specifying the model are (for each family) the average comoving distance between meatballs $d_{c}$, the proper radius of the meatball $R_{p}$ and the mass of the meatball $M$. The numerical values we used are given in Table 1

The distance $d_{c}$ between meatballs is connected to their comoving number density by $n_{c}=\frac{\Gamma(4 / 3)^{3}}{(4 \pi / 3)} d_{c}^{-3}$. We assume the meatballs to have virialized at $z=1.6$ and therefore their proper radii $R_{p}$ are constants. For the family $B$ we used the singular isothermal spheres (SIS) density profile and defined the radius to have a density contrast of 200 at virialization. For the family $A$ the gaussian profile was used and the radius was chosen to qualitatively describe the largest structures seen in simulations of structure formation.

\footnotetext{
${ }^{1}$ Contribution to the proceedings of the Invisible Universe International Conference, Paris, France, June 29-July 3, 2009. Based on [1, 2], where more details and references can be found.
} 
TABLE 1. Parameters of the meatball model $\left(a_{0} \equiv 1\right)$.

\begin{tabular}{lcc}
\hline Quantity & Family $A$ & Family $B$ \\
\hline$d_{c}$ & $100 h^{-1} \mathrm{Mpc}$ & $10 h^{-1} \mathrm{Mpc}$ \\
$R_{p}$ & $10 h^{-1} \mathrm{Mpc}$ & $580 h^{-1} \mathrm{kpc}$ \\
$M$ & $6.1 \cdot 10^{17} h^{-1} M_{\odot}$ & $6.1 \cdot 10^{14} h^{-1} M_{\odot}$ \\
density profile & Gaussian & $\mathrm{SIS}$ \\
\hline
\end{tabular}

The distribution of family $A$ meatballs leaves underdense regions of order $100 h^{-1} \mathrm{Mpc}$ which are filled by the family $B$ meatballs. Because the mass is equally subdivided between the two families, these underdense regions have on average a density contrast of $\delta \approx-0.5$. The second element of our setup consists of placing the observer in one of the large underdense regions and modelling the metric of this particular void around the observer more accurately with a Lemaitre-Tolman-Bondi (LTB) bubble matched to the EdS background metric. See Fig. 11 for a sketch.

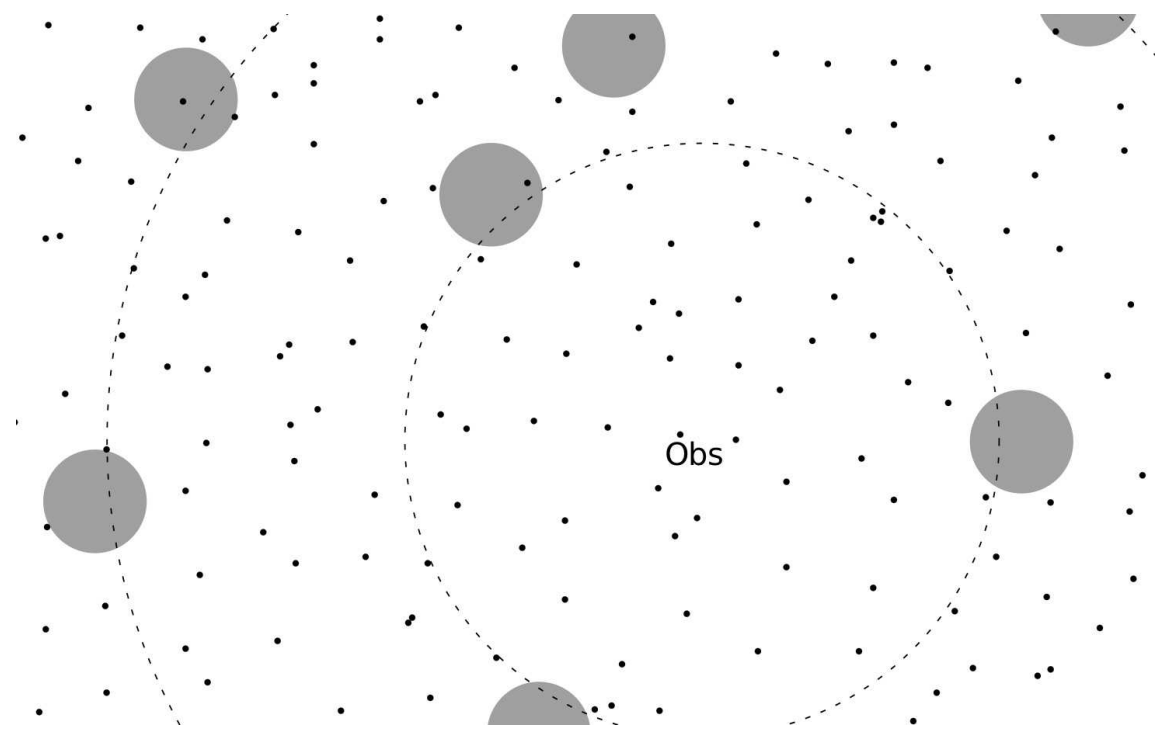

FIGURE 1. The larger disks represent the meatballs of the family $A$, while the smaller ones represent the meatballs of the family $B$. The concentric circles mark the overdense shells in the LTB bubble and are roughly $100 h^{-1} \mathrm{Mpc}$ apart.

\section{LENSING}

In a late-time universe dominated by voids the homogeneity is recovered only on scales larger than the largest inhomogeneity scale, which in our model is $L_{\text {hom }} \sim 100 h^{-1} \mathrm{Mpc}$. However, type Ia supernovae (SNe) probe angular scales $L_{S N e} \ll L_{h o m}$ and it is therefore not clear that the physics inferred from SNe observations can be directly associated with the smoothed-out GBS model, especially with small data samples. Epigrammatically, the commutativity between averaging and measuring is not guaranteed. Note that this idea belongs to weak-backreaction studies and is different from the possible non-commutativity between averaging and dynamics which is the kernel of strong-backreaction studies (see for example Ref. [6]):

$$
\begin{aligned}
& {[\langle\cdots\rangle, \mathrm{EoM}] \neq 0 \Rightarrow \mathrm{ABS} \neq \mathrm{GBS}} \\
& {[\langle\cdots\rangle, \text { Obs }] \neq 0 \Rightarrow \text { PBS } \neq \text { GBS }}
\end{aligned}
$$

See [3] for more details about a proposed framework for the backreaction proposal.

Cumulative gravitational lensing is a possible source for the non-commutativity between averaging and measuring and to study this effect we have focused on a meatball model. The latter indeed incorporates quantitatively the crucial feature that photons can travel through voids missing the localised overdensities. This feature, instead, is not present in swiss-cheese models where the bubble boundaries are designed to have compensating overdensities. Such models have indeed been shown to have on average little lensing effects [7]. 


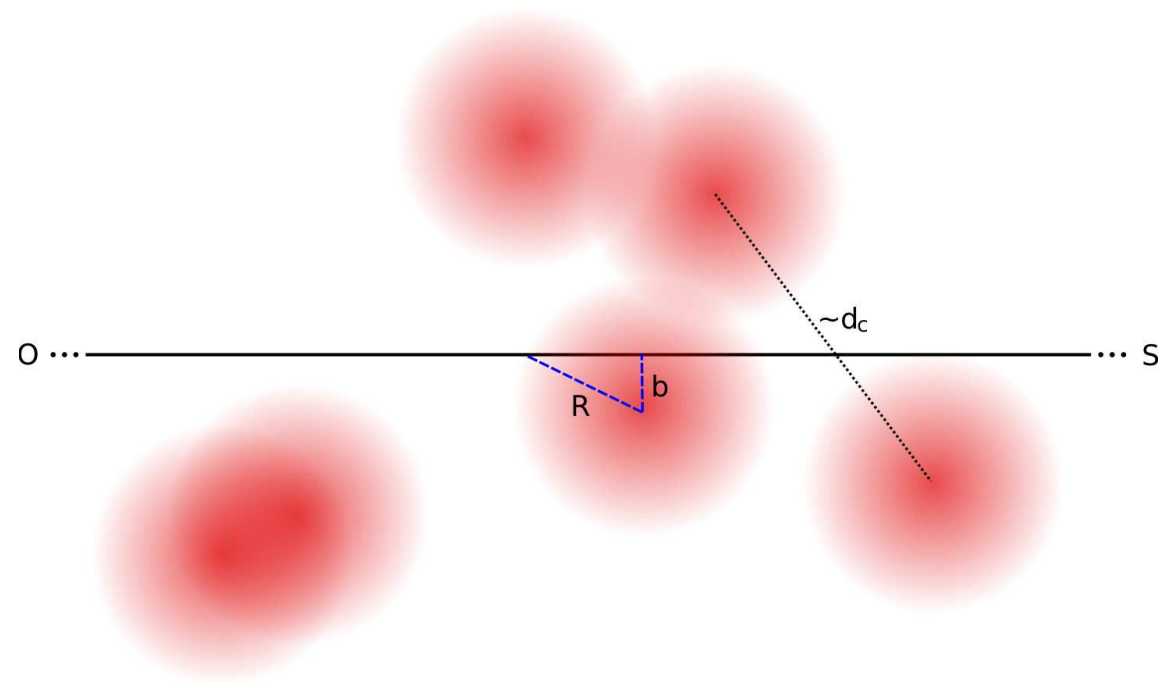

FIGURE 2. Shown is a co-moving segment of a photon geodesic between an observer at $O$ and a source at $S$. The shaded disks represent meatballs of radius $R$.

In the weak-lensing approximation the lens convergence $\kappa$ is given by

$$
\kappa(z)=\int_{0}^{r_{s}(z)} d r G\left(r, r_{s}(z)\right) \delta(r, t(r))
$$

where $\delta(r, t)$ is the density contrast and

$$
G\left(r, r_{s}\right)=\frac{3 H_{\infty}^{2}}{2 c^{2}} \frac{r\left(r_{s}-r\right)}{r_{s}} \frac{1}{a(t(r))}
$$

The functions $a(t), t(r)$ and $r(z)$ correspond to the EdS background model, $r_{s}$ is the comoving position of the source at redshift $z$ and the integral is evaluated along the unperturbed light path. We will denote by $\kappa_{E}$ the convergence of the "empty beam" which is given by Eq. (1) with $\delta=-1$. The shift in the distance modulus, neglecting the second-order contribution of the shear, is

$$
\Delta m(z)=5 \log _{10}(1-\kappa(z)) .
$$

$\Delta m$ is proportional to the total matter column density along the photon path. For a lower-than-GBS column density the light is demagnified, while in the opposite case it is magnified.

In Ref. [2] we derived a fast and easy way to obtain the convergence probability distribution function (PDF) for meatball models. First define a normalized profile $\varphi(x)=\rho(x) / \bar{\rho}$, where $\rho$ is the density profile of a meatball and $\bar{\rho}$ is the EdS density. Then the following quantity characterizes the contribution from one meatball, hit with an impact parameter $b$, to the convergence (see Fig. 2 for an illustration of the calculation):

$$
\Gamma(b, t)=\int_{b}^{R(t)} \frac{2 x d x}{\sqrt{x^{2}-b^{2}}} \varphi(x, t) .
$$

Then divide the comoving distance $r_{s}$ to the source and the radius $R$ of the meatball into $N_{S}$ and $N_{R}$ bins of widths $\Delta r_{i}$ and $\Delta b_{m}$, such that $\Delta b_{m} \ll R$ and $R \ll \Delta r_{i} \ll r_{s}$. The convergence due to a meatball placed within the bin $(i, m)$ is just

$$
\kappa_{1 i m}=G\left(r_{i}, r_{s}\right) \Gamma\left(b_{m}, t_{i}\right) .
$$

The PDF is then generated by the following functional

$$
\kappa\left(\left\{k_{i m}\right\}\right)=\sum_{i=1}^{N_{S}} \sum_{m=1}^{N_{R}} \kappa_{1 i m}\left(\frac{k\left[N_{O} \Delta N\right]_{i m}}{N_{O}}-\Delta N_{i m}\right),
$$


where $k\left[N_{O} \Delta N\right]_{i m}$ is a Poisson random variable of parameter $N_{O} \Delta N_{i m} . N_{O}(z)$ is the number of observations at redshift $z$ and $\Delta N_{i m}=n_{c} \Delta V_{i m}$ is the expected number of meatballs in the volume $\Delta V_{i m}=2 \pi b_{m} \Delta b_{m} \Delta r_{i}$. For a Poisson variable of parameter $\lambda$, the mean is $\lambda$ and so the expected convergence in Eq. (6) is zero, consistent with photon conservation in weak lensing.

The PDF is generated by creating a large sample of configurations $\left\{k_{i m}\right\}$ drawn from the Poisson distribution, and computing the appropriate convergences through Eq. (6). This is a task that a laptop fullfils in less than a second as opposed to expensive ray tracing techniques. The weak lensing approximation of Eq. (6) describes the mode of the PDF very well always and, in case of non-point-like meatballs, also the tails of the PDF with $<5 \%$ of error. See Fig. 3 for an example relative to the present model.

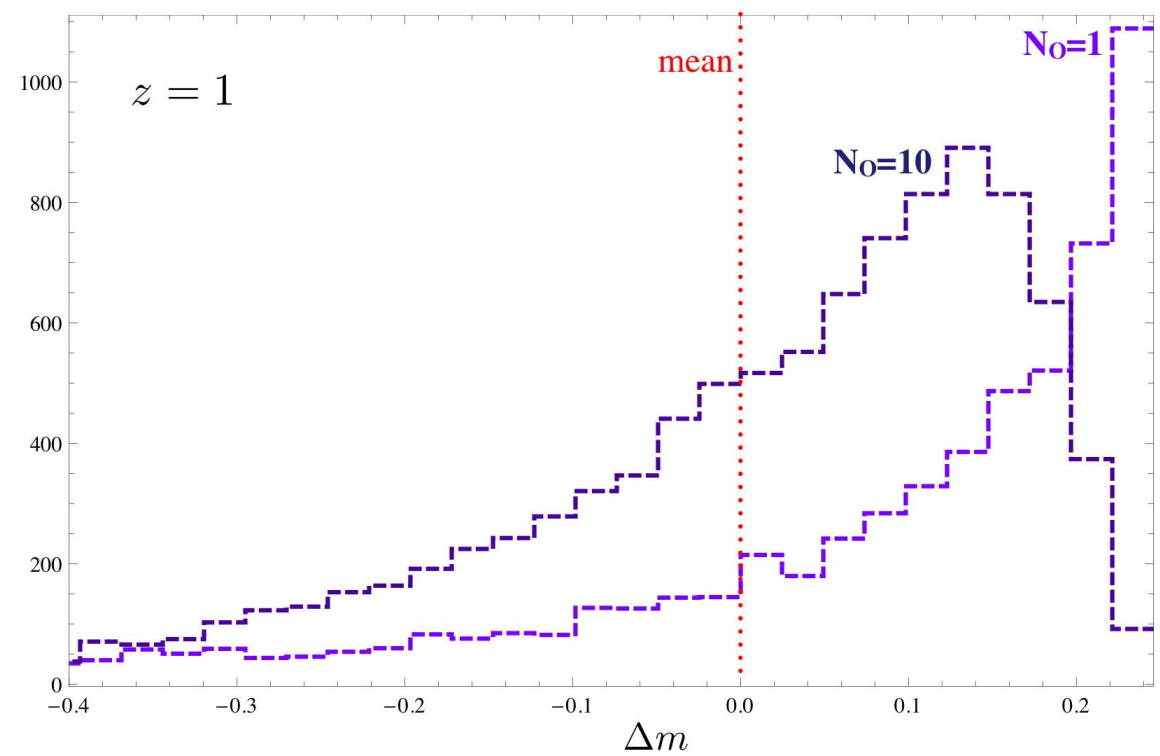

FIGURE 3. Lensing PDF in magnitudes for the meatball model of this paper at redshift $z=1$ and for a set of 1 and $10 \mathrm{SNe}$. Increasing the number of measurements reduces the skewness of the PDF, which, however, is still highly non gaussian after 10 measurements.

Eq. (6) displays explicitly the effect of the size of the data sample: even if $\kappa$ might have a skewed PDF and a nonzero mode for $N_{O}=1$, the gaussianity is recovered for large $N_{O}$ and the set of observations becomes unbiased. To define $N_{O}(z)$ we will use the Union Catalogue of Ref. [8], which consists of 307 SNe spread between $0<z<1.6$. We have binned the SNe with a bin width of $\Delta z=0.1$ and the result in plotted in the top panel of Fig. 4. SNe observations within the same bin are then be treated as repetition of the same observation.

An analytical approximation for the PDF was found in Ref. [2] by resumming the individual Poisson varaibles. The result is simply

$$
\kappa(k)=\kappa_{E}(z)\left(1-\frac{k\left[N_{O} N\right]}{N_{O} N}\right),
$$

where $k$ is again a Poisson random variable and

$$
N=n_{c} \cdot r_{s}(z) E \cdot \pi \bar{R}^{2} Q_{\varphi}^{2}
$$

Physically $N$ corresponds to the expected number of collisions with metaballs in a tube of a radius $\bar{R} Q_{\varphi}$ and a length $r_{S} E$ connecting the observer to the source. The constants $E \simeq 2 / 3$ and $Q_{\varphi}$ are corrections for the effective comoving distance and the effective meatball radius. For the gaussian meatballs of the first family $Q_{\varphi}^{A} \simeq 0.5$ and for the SIS meatballs of the second family $Q_{\varphi}^{B} \simeq 0.25$. The co-moving size $\bar{R}$ is computed at half distance between observer and source. Eq. (7) reproduces the results of Eq. (6) quite well for a wide range of parameters, and so Eq. (8) provides an easy way to estimate the magnification bias. If meatballs fill the universe smoothly (large $n_{c}$ ) or their density profile is wide and smooth (large $\bar{R}$ ) then $N$ is large leaving a negligible magnification bias. Moreover, even for a small $N$ a large $N_{O}$ removes the non-Gaussianity from the effective PDF. However, if the product $N_{O} N$ is small, the lens convergence approaches the empty beam limit as the light rays "scan" mostly voids. This gives rise to a low effective column density and therefore to an average demagnification. For a thorough analysis of the previous results we refer to Ref. [2]. 


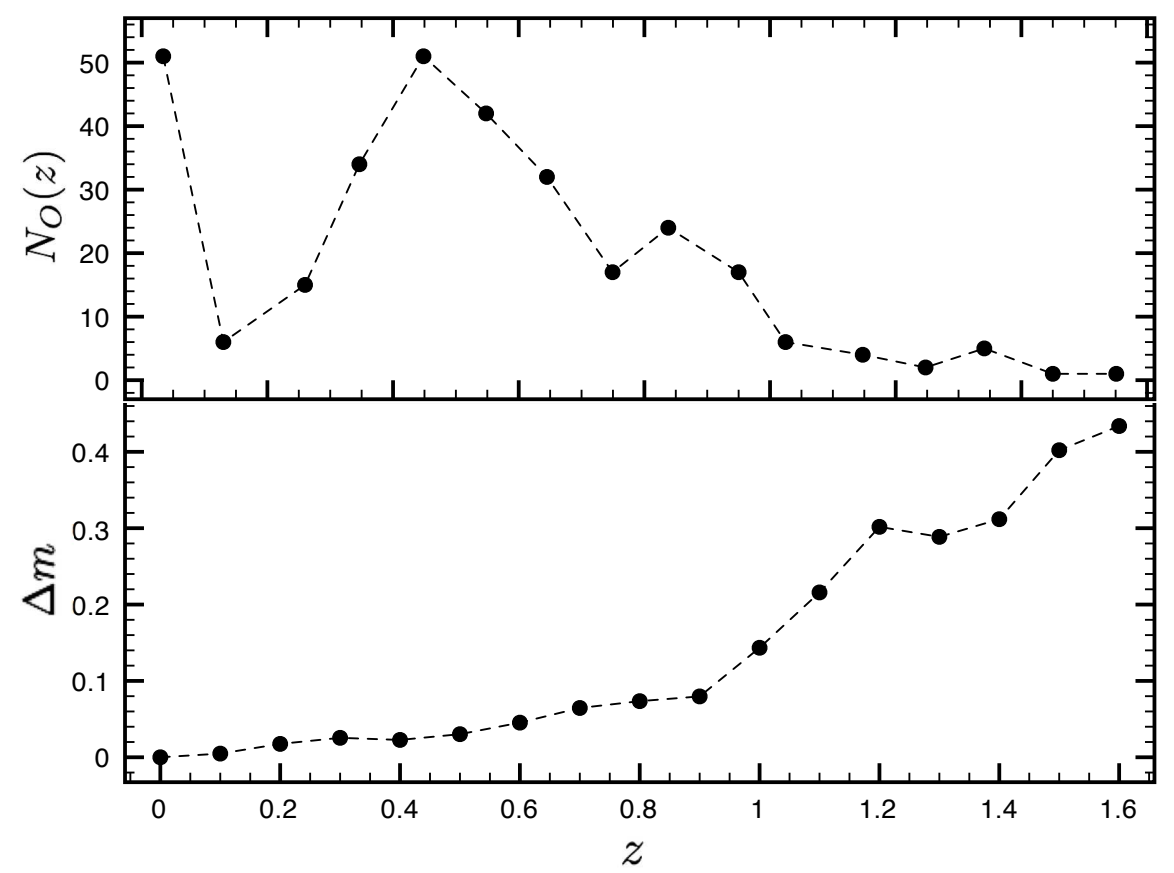

FIGURE 4. Top: the $307 \mathrm{SNe}$ of the Union Catalogue of Ref. [8] binned with $\Delta z=0.1$. Bottom: shift in the distance modulus $\Delta m$ for $N_{O}$ taken from the top panel and for the meatball model of this paper.

We have used a modification of the turboGL package [9] to evaluate the magnification bias for our model and for the Union Catalogue. Results are shown in the bottom panel of Fig. 4 The magnification bias is nonneglible for $z \geq 0.5$ and large for $z \geq 1$.

\section{HUBBLE BUBBLE}

The second element of our model consists of using an LTB bubble matched to the EdS background metric of the meatball model to describe the local metric around the observer (see Ref. [10] for a discussion about matching in swiss-cheese models). The new ingredient is that an observer inside a void expanding faster than the background sees an apparent acceleration (see, e.g., Refs. [11, 12]). This effect is easy to understand: our cosmological observables are confined to the light cone and hence temporal changes can be associated with spatial changes along photon geodesics. For example "faster expansion now than before" is simply replaced by "faster expansion here than there". This is why a local hubble bubble model can mimic the effect of a cosmological constant at recent times $(z \leq 1)$.

The price to pay in a simple hubble bubble model is that the inhomogeneity has to extend to the point in space/time where the effect of dark energy disappears. This requires [12] an enormous local void of radius $1.5-2 h^{-1} \mathrm{Gpc}$. Moreover, to avoid a too large dipole in the CMB, our position in the void would have to be very special leading to a gross violation of the Copernican principle. The model of this letter features inhomogeneities on much smaller scales. Here the effects of the dark energy at large redshift $(z \geq 0.5)$ are mimicked by the lensing bias, and the local void only needs to model the cosmological constant at small redshifts where the lensing effects are neglible.

The spherically symmetric dust LTB metric can be written as

$$
d s^{2}=-c^{2} d t^{2}+\frac{R^{\prime 2}(r, t)}{1-k(r) r^{2}} d r^{2}+R^{2}(r, t) d \Omega^{2} .
$$

This reduces to the usual FLRW metric when $R(r, t) / r \equiv a(r, t) \rightarrow a(t)$ and $k(r) \rightarrow \pm 1$ or 0 . The Einstein equation for this metric is

$$
\frac{\dot{a}^{2}(r, t)}{a^{2}(r, t)}=\frac{8 \pi G}{3} \hat{\rho}(r, t)-\frac{c^{2} k(r)}{a^{2}(r, t)},
$$




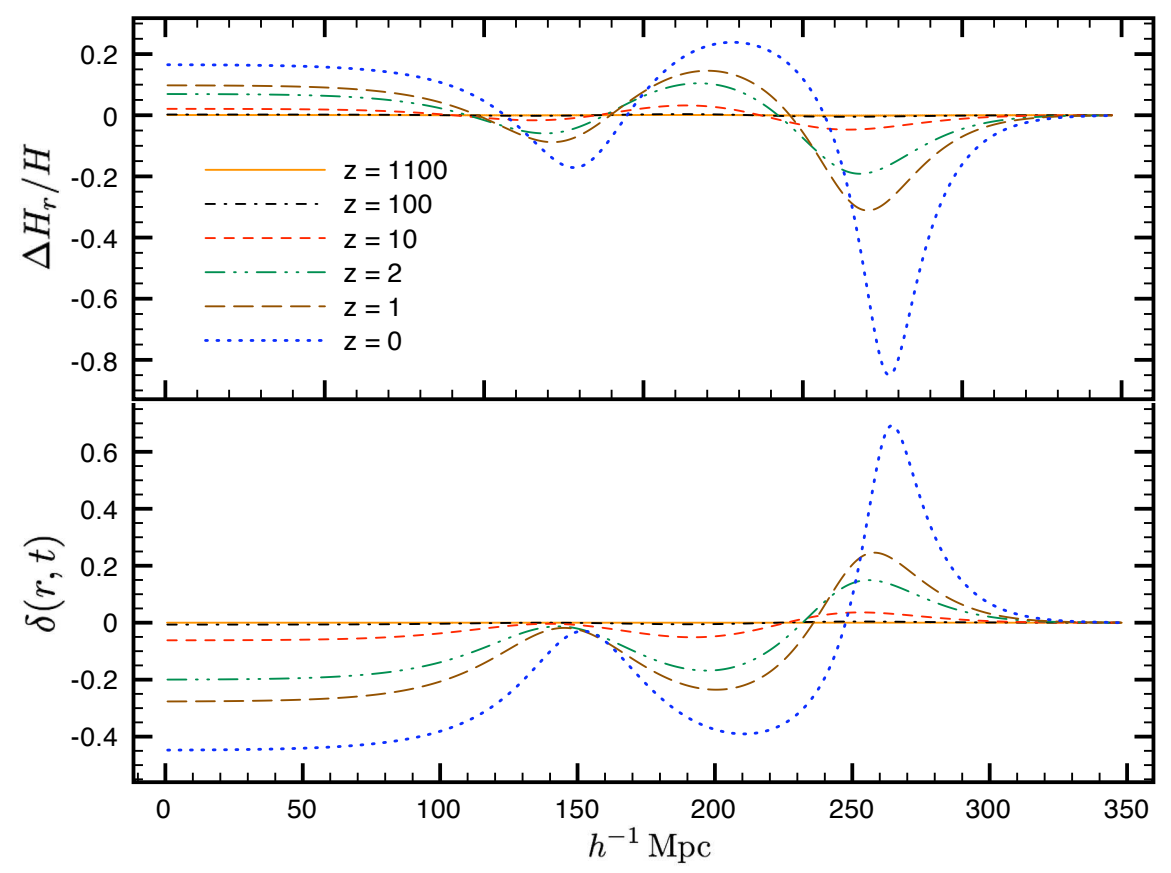

FIGURE 5. $\Delta H_{r} / H$ (top panel) and density contrast (bottom panel) with respect to normalized proper distance $(R / a(t))$ at times corresponding to the redshifts indicated in the plot.

where $\hat{\rho}$ is the average density up to the shell $r$. We specify our LTB model at the recombination time with a density contrast of order $\sim 10^{-3}$ in accordance with the CMB. The subsequent evolution of the density contrast is shown in Fig. 5. Also shown is the ratio $\Delta H_{r} / H$ where $\Delta H_{r}=H_{r}-H, H$ is the EdS expansion rate and $H_{r}=\dot{R}^{\prime} / R^{\prime}$ is the radial expansion rate in the LTB bubble. At late times the initial perturbations generate two fast expanding $\left(\Delta H_{r}>0\right)$ voids of radius $50-100 h^{-1} \mathrm{Mpc}$, surrounded by two thin collapsing $\left(\Delta H_{r}<0\right)$ shells. The inner void of radius $\sim 100 h^{-1} \mathrm{Mpc}$, where the observer is located, has a local expansion rate of $h \simeq 0.58$ which, as anticipated, is larger than the EdS value. Moreover, the density contrast of the latter is close to $\delta \approx-0.5$ consistently with our meatball model for the overall universe.

In order not to have a too large dipole in the CMB the observer has to be at a distance $\leq 15 h^{-1} \mathrm{Mpc}$ from the center of the inner void. Tighter constrains will come from future probes sensitive to off-center anisotropy (see, for example, [13]).

\section{RESULTS}

Let us now describe the main features of our results shown in Fig. 6. First, a matter dominated model cannot exhibit local acceleration, as one can formally show using the Raychaudhuri's equation [6]. This behaviour is evident in the zoom in the bottom panel, where the slope of the inhomogenous model curve is seen to start flat at $z=0$. However, at finite redshifts the spatial variation of the expansion rate mimics the apparent acceleration and a positive $\Delta m$ proportional to the difference between local and background expansion rates $H_{0}-H_{\infty}$ appears. Beyond the dimensions of the local hubble bubble, at $z \geq 0.1$, the slope of the curve follows the EdS model, until the lensing bias effect becomes effective at $z \geq 0.5$ and starts to pull the curve up again as is clearly seen in the top panel in fig.6.

We performed also a $\chi^{2}$ analysis to estimate the goodness-of-fit of our model. Results are shown in Table 2. Obviously the EdS model has a poor fit, but introducing inhomogeneities improves the $\chi^{2}$ significantly. The local Hubble bubble has a larger impact on $\chi^{2}$, but lensing also has a large positive effect. Our model is still worse than the $\Lambda$ CDM model, but not dramatically so. The main characteristic differences between our inhomogenous model and the $\Lambda \mathrm{CDM}$ model are the smooth rise of the predicted $\Delta m$ at large $z$ in the former model and the different behaviour around $z \sim 0.1-0.2$, as seen in Figure 6 Both these issues would be easily resolved by a JDEM-like survey with a data set of $2000 \mathrm{SNe}$. 


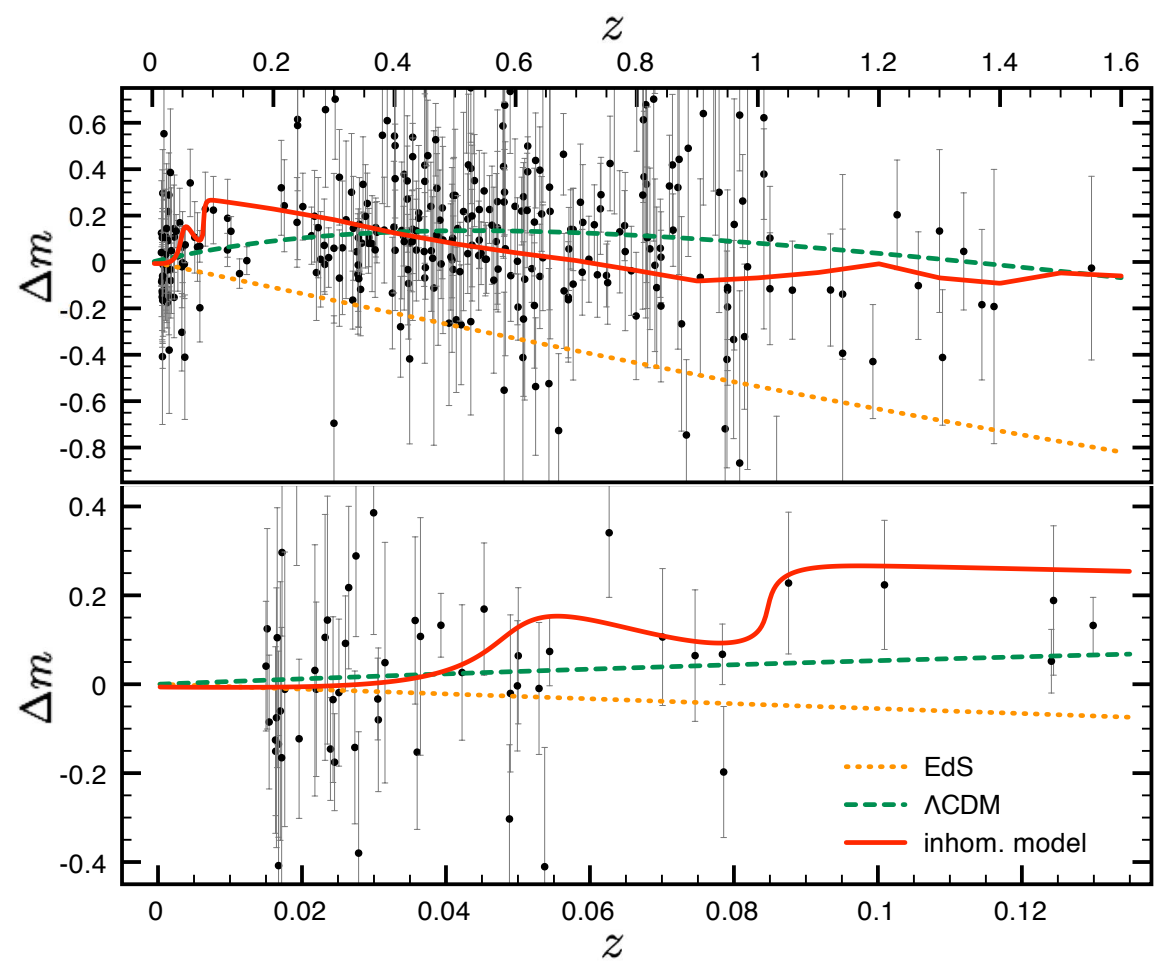

FIGURE 6. Top panel: shown is the distance modulus with respect to the empty universe for the inhomogeneous model of this paper, for the $\Lambda \mathrm{CDM}$ model and for the EdS model together with the full Union Catalogue of Ref. [8]. Bottom panel, zoom for small redshifts.

We would like to stress that we did not explore all the parameter space in order to minimize the $\chi^{2}$. We indeed chose, as explained before, the parameters qualitatively and we calculated the $\chi^{2}$ to give a goodness-of-fit of the meatball model with respect to the concordance model.

Finally let us note that including inhomogeneities changes also the predictions of the $\Lambda$ CDM model. In Table 2 we give the result of a simulation for the same meatball mass spectrum we used with the EdS model, in the context of a $\Lambda \mathrm{CDM}$ model whose global parameters are set to those used in Ref. [8]. Interestingly, including inhomogeneities to the concordance model makes the fit worse and suggests, as discussed in Ref. [14], a smaller value of $\Omega_{\Lambda}$.

TABLE 2. $\chi^{2}$ for Union Catalogue.

\begin{tabular}{lc}
\hline Model & $\chi^{2}$ \\
\hline$\Lambda \mathrm{CDM}$ & 312 \\
$\Lambda \mathrm{CDM}+$ meatballs & 323 \\
EdS & 608 \\
EdS + H. bubble & 440 \\
EdS + H. bubble + meatballs & 396 \\
\hline
\end{tabular}

Of course our setup is but a simple toy model. However, we believe that our results make it explicitly clear that different effects of inhomogeneities can pull into the same direction, making the PBS depart from the GBS. Moreover, we believe that the two effects of the voids considered here are not the end of the story; to conclude on the viability of inhomogeneous universe models as the possible explanation of the apparent acceleration, it is crucial to consider all possible effects in as realistic a model as possible.

\section{REFERENCES}

1. K. Kainulainen and V. Marra, arXiv:0906.3871 [astro-ph.CO], accepted for publication in Phys. Rev. D.

2. K. Kainulainen and V. Marra, arXiv:0909.0822 [astro-ph.CO]. 
3. E. W. Kolb, V. Marra and S. Matarrese, Gen. Rel. Grav. (2009), arXiv:0901.4566 [astro-ph.CO].

4. W. L. Freedman et al., Astrophys. J. 553, 47 (2001).

5. The most recent limit is $h=0.742 \pm 0.036$ from A. G. Riess et al., Astrophys. J. 699, 539 (2009).

6. G. F. R. Ellis, in Gen. Rel. and Grav., Reidel, Dordrecht, 1984, p. 215; T. Buchert, Gen. Rel. Grav. 40, 467 (2008); E. W. Kolb, S. Matarrese and A. Riotto, New J. Phys. 8, 322 (2006); A. A. Coley, N. Pelavas and R. M. Zalaletdinov, Phys. Rev. Lett. 95, 151102 (2005); D. L. Wiltshire, New J. Phys. 9, 377 (2007); E. W. Kolb, V. Marra and S. Matarrese, Phys. Rev. D 78, 103002 (2008).

7. N. Brouzakis, N. Tetradis and E. Tzavara, JCAP 0804, 008 (2008); R. A. Vanderveld, E. E. Flanagan and I. Wasserman, Phys. Rev. D 78, 083511 (2008); W. Valkenburg, JCAP 0906, 010 (2009).

8. M. Kowalski et al., Astrophys. J. 686, 749 (2008).

9. turboGL is available at: http://www.turbogl.org

10. V. Marra, E. W. Kolb, S. Matarrese and A. Riotto, Phys. Rev. D 76, 123004 (2007); V. Marra, E. W. Kolb and S. Matarrese, Phys. Rev. D 77, 023003 (2008); V. Marra, Padua@ research ID588, arXiv:0803.3152 [astro-ph].

11. N. Mustapha, C. Hellaby and G. F. R. Ellis, Mon. Not. Roy. Astron. Soc. 292, 817 (1997); M. N. Célérier, Astron. Astrophys. 353, 63 (2000); K. Tomita, Mon. Not. Roy. Astron. Soc. 326, 287 (2001); H. Iguchi, T. Nakamura and K. i. Nakao, Prog. Theor. Phys. 108, 809 (2002); D. J. H. Chung and A. E. Romano, Phys. Rev. D 74, 103507 (2006); K. Enqvist and T. Mattsson, JCAP 0702, 019 (2007); S. Alexander, T. Biswas, A. Notari and D. Vaid, JCAP 0909, 025 (2009).

12. H. Alnes, M. Amarzguioui, and O. Grøn, Phys. Rev. D 73, 083519 (2006); J. Garcia-Bellido and T. Haugboelle, JCAP 0909, 028 (2009); J. P. Zibin, A. Moss and D. Scott, Phys. Rev. Lett. 101, 251303 (2008).

13. M. Quartin and L. Amendola, arXiv:0909.4954 [astro-ph.CO].

14. T. Clifton and P. G. Ferreira, JCAP 0910, 26 (2009). 